\title{
Convergence in a neo-Kaleckian model with endogenous technical progress and autonomous demand growth*
}

\author{
Won Jun $\mathrm{Nah}^{\star *}$ \\ Associate Professor, School of Economics and Trade, Kyungpook National University, Daegu, Korea \\ Marc Lavoie ${ }^{\star * *}$ \\ Senior Research Chair, University of Sorbonne Paris Cité (University of Paris 13, CEPN), France and \\ Emeritus Professor, Department of Economics, University of Ottawa, ON, Canada
}

This paper introduces technical progress along the lines of the Kaldor-Verdoorn law within a neo-Kaleckian model of growth and distribution that incorporates the Sraffian supermultiplier mechanism. The key features of the model include the interactive effects of endogenous technical progress, the non-capacity-creating demand component that grows at an exogenous rate and, in its long-run version, a Harrodian adjustment mechanism. It turns out that, whereas the model converges towards the normal rate of capacity utilization, the main tenets of the Keynesian model are still valid in the long run as well as in the short run in the sense that all of the average rates of accumulation, capacity utilization, and technical progress are lower during the traverse after the propensity to save or the share of profits goes up. The conditions under which the productivity regime can be wage-led are examined, and the possible effects of an exogenous technical shift are also discussed.

Keywords: neo-Kaleckian, growth, capacity utilization, technical progress, autonomous expenditures

JEL codes: $E 11, F 41, O 41$

\section{INTRODUCTION}

Even with all the bustle of 'the 4th industrial revolution,' the recent decade has witnessed an apparent slowdown in productivity growth. In the United States, for instance, the annual rate of growth of labor productivity has been around 1.3 percent on average since 2007. Since 2010, it has become even lower, at 0.5 percent. It goes without saying that these figures are in stark contrast to the one corresponding to the Golden Age of capitalism, which amounted to 2.8 percent for a 26-year average. A couple of different explanations for the recent stagnation have been put forth, and one of them is based on the observation that the productivity slowdown is a by-product of the Great Recession

* We thank the referees for their useful advice. The paper was presented in February 2017 at a seminar at Kingston University, UK, and at a meeting of the Korean Association for Political Economy, Korea.

** Email: wjnah@knu.ac.kr.

*** Email: mlavoie@uottawa.ca. 
in the aftermath of the global financial crisis. With demand depressed and the expected growth rate of sales falling, firms can be reluctant to expand productive capacity, and defer or even cancel the introduction of new technologies into production processes, resulting in a productivity slowdown, as in the so-called Kaldor-Verdoorn law (McCombie 2002).

The concept of the Kaldor-Verdoorn law has been provided recently with more empirical support. Reifschneider et al. (2015) argue that the sustained drop in productivity can be the result of a decline in investments, which implies that the recent productivity slowdown is an endogenous response to the contraction in economic activity. Anzoategui et al. (2019) embed the mechanism of the costly adoption of new technologies into a New Keynesian model and make the case that the fall in productivity following the Great Recession was an endogenous phenomenon reflecting the contraction in aggregate demand. In a similar vein, Mason (2017) emphasizes that the slow productivity growth in the recent decade can be understood as the result of weak demand.

The purpose of the present paper is to introduce technical progress along the lines of the Kaldor-Verdoorn law within a neo-Kaleckian model of growth and distribution that incorporates a non-capacity-creating demand component that grows at an exogenous rate. There has been a recent revival of interest among post-Keynesians for the role that this autonomous component of aggregate demand could play in the economy, arising from two strands. First, there are models that extend the arguments put forward by Serrano (1995a; 1995b) and by Bortis (1997) under the name of the Sraffian supermultiplier, as can be found in the recent works of Cesaratto (2015), Freitas and Serrano (2015), Pariboni (2016a), Dejuán (2017), and Serrano and Freitas (2017). Cesaratto et al. (2003) discuss the role of technical progress in this kind of model. Second, there are models that incorporate the autonomous growth component within a neo-Kaleckian model of growth and distribution, as can be found in the papers of Lavoie (2014; 2016), Allain $(2015 ; 2019)$, Dutt (2015; 2019), Pariboni (2016b), Nah and Lavoie (2017; 2018; 2019), and Hein (2018). It is this second strand which is at the heart of the present paper.

The present work starts from Lavoie (2016), who shows how one can introduce an exogenously growing component of consumption into a neo-Kaleckian model of growth and distribution, with overall growth converging to the growth rate of exogenous demand. In this paper, we focus on the interactions between endogenous technical progress, the autonomous demand component, and a Harrodian instability mechanism. While the autonomous component of demand ultimately determines the long-run growth rate, endogenous technical progress amplifies the growth impact of capital accumulation in the traverse because faster capital accumulation raises the rate of technical progress. In addition, the paper endogenizes the parameters of the investment function so that the desired rate of accumulation responds to the actual rate of growth. It turns out that with this addition the model converges towards the normal rate of capacity utilization in the long run, but the main tenets of the Keynesian model are still valid in the short, medium, and long run, in the sense that the average rates of accumulation, capacity utilization, and technical progress are lower when we consider the whole duration of adjustments responding to an increase in the propensity to save or in the share of profits. Also we have examined the conditions under which the productivity regime can be wage-led, and long-run employment can be demand-determined.

We shall proceed as follows. In Section 2 we present the key elements of the model. Section 3 and 4 discuss the short-run and medium-run solutions respectively of the model. Section 5 discusses long-run solutions when the investment function incorporates a Harrodian mechanism. In all these sections we examine what happens if there is a 
change in some of the parameters, in particular the propensity to save, the share of profit, or the growth rate of the exogenous non-capacity-creating component of aggregate demand. In Section 6 we focus on the consequences of a change in the exogenous component of the growth rate of technical progress. Section 7 deals with the consequences of such a change for employment and Section 8 concludes.

\section{THE ECONOMIC ENVIRONMENT}

We extend a version of the neo-Kaleckian model of growth found in Lavoie (2016), by adding to it considerations linked to an endogenous technical progress function. Our model will ultimately encompass the interactive effects of technical progress, the autonomous growth of a component of expenditures, and a Harrodian investment function. For simplification, we consider a closed economy without a public sector.

The model economy is populated by capitalists who own capital and workers who do not. The functional income distribution between these two income groups is assumed to be determined by political and institutional factors, and hence assumed in the model to be exogenously given. The rate of profit, the rate of utilization of capacity and the rate of accumulation of capital, along with the rate of technical progress, will be endogenously determined.

We deal with only Harrod-neutral technical progress, which is capital-embodied and concomitant with a decrease in the labor-to-output ratio. To be specific, technical progress in our model is assumed to be described by the standard Kaldor-Verdoon equation, as can be found in Kaldor (1978), which can be interpreted as a variant of his technical progress function (Kaldor 1957).

$$
\lambda=\lambda_{0}+\lambda_{g} g,
$$

where $\lambda_{0}>0$ and $\lambda_{g}>0$. Here, $\lambda$ denotes the growth rate of output per labor, and $g$ the rate of accumulation. This specification postulates that the growth rate of labor productivity is positively associated with economic expansion due to dynamic increasing returns.

Let us assume that a part of capitalists' consumption is an autonomous component $Z$ and hence that the aggregate consumption of capitalists becomes

$$
Z+\left(1-s_{p}\right) P
$$

where $s_{p}$ is the marginal propensity to save of capitalists and $P$ stands for profits. With this kind of consumption by capitalists, and under the assumption that workers do not save and that there is no depreciation, the saving function can be specified as follows:

$$
\sigma=s_{p} \pi u-z
$$

where $\sigma$ is capitalists' saving normalized by the capital stock $K, \pi$ the share of profits out of national income, $u$ the ratio of output to capital which is the proxy for the rate of utilization of capacity for a given $K$, and $z=Z / K$.

For the investment function, we employ the following specification:

$$
g=\gamma+\gamma_{u}\left(u-u_{n}\right)+\gamma_{\lambda} \lambda
$$

where $g$ is the rate of accumulation, $\gamma$ the variable which captures the effects of animal spirits, $u_{n}$ the normal or the target rate of capacity utilization, and with $\gamma_{u}>0$ and $\gamma_{\lambda}>0$. This specification explicitly assumes that the rate of technical progress 
increases the rate of accumulation, other things being equal, as can be found in previous neo-Kaleckian models such as those of Rowthorn (1981, p. 23) and Lavoie (1992, p. 318). As long as productivity growth is fully embodied in the stock of capital, capitalists facing higher technical progress will increase the pace of investment in an attempt to gain from it. In other words, the pace of innovation is a shift parameter of the Kaleckian investment function. As recalled by Dutt (1990, p. 106), this is an argument that was made earlier by Kalecki (1971, ch. 15), and it was also supported by Steindl $(1979$, p. 7). There is also quite a lot of similarity with the views endorsed by neo-Schumpeterians, who contend that firms and their bankers ride on waves of innovation so that technical progress ought to have a positive effect on investment.

Plugging (1) into (3), we have:

$$
g=\gamma+\gamma_{u}\left(u-u_{n}\right)+\gamma_{\lambda}\left(\lambda_{0}+\lambda_{g} g\right) .
$$

Solving for $g$, we get:

$$
g=\frac{\gamma-\gamma_{u} u_{n}+\gamma_{\lambda} \lambda_{0}}{1-\gamma_{\lambda} \lambda_{g}}+\frac{\gamma_{u}}{1-\gamma_{\lambda} \lambda_{g}} u
$$

\section{SHORT-RUN EQUILIBRIUM}

The basic characteristics of model dynamics can easily be seen with the help of the notion of short-run equilibrium, where saving adjusts to investment. In the model, the condition for short-run equilibrium, where both $z$ and $\gamma$ are constant, is given by

$$
\dot{u}=0 \text {, }
$$

with $\dot{u}=\phi_{0}(g-\sigma)$ and $\phi_{0}>0$. This implies:

$$
\begin{gathered}
u^{*}=\frac{\gamma-\gamma_{u} u_{n}+\gamma_{\lambda} \lambda_{0}+z\left(1-\gamma_{\lambda} \lambda_{g}\right)}{s_{p} \pi\left(1-\gamma_{\lambda} \lambda_{g}\right)-\gamma_{u}} \\
g^{*}=\frac{\gamma-\gamma_{u} u_{n}+\gamma_{\lambda} \lambda_{0}}{1-\gamma_{\lambda} \lambda_{g}}+\frac{\gamma_{u}}{1-\gamma_{\lambda} \lambda_{g}} u^{*} \\
\lambda^{*}=\lambda_{0}+\lambda_{g} g^{*},
\end{gathered}
$$

where * denotes the value of a variable in short-run equilibrium. Note that, as is standard in neo-Kaleckian models, we assume the following Keynesian stability condition to hold:

$$
s_{p} \pi\left(1-\gamma_{\lambda} \lambda_{g}\right)>\gamma_{u}
$$

Since $\gamma_{u}>0$, a necessary but not sufficient condition for inequality (9) to hold is that $\gamma_{\lambda} \lambda_{g}<1$.

It is straightforward to check that an increase in the marginal propensity to save, $s_{p}$, results in decreases in $u^{*}$ and $g^{*}$ in the short run. This confirms the paradox of thrift, which is a well-known result in the Keynesian/Kaleckian literature. Also a change in functional income distribution in favor of capital, to the detriment of labor, decreases 
$u^{*}$ and $g^{*}$ in the short run. This means that the present model generates wage-led, instead of profit-led, demand and growth regimes in the short run. The negative effects of an increase in the profit share on demand and growth are exerted via consumption demand:

$$
\begin{gathered}
\frac{\partial u^{*}}{\partial \pi}=-s_{p} \cdot \frac{\left(1-\gamma_{\lambda} \lambda_{g}\right) u^{*}}{s_{p} \pi\left(1-\gamma_{\lambda} \lambda_{g}\right)-\gamma_{u}}<0 \\
\frac{\partial g^{*}}{\partial \pi}=\frac{\gamma_{u}}{1-\gamma_{\lambda} \lambda_{g}} \frac{\partial u^{*}}{\partial \pi}<0 .
\end{gathered}
$$

In our model, the growth rate of labor productivity is positively affected by the rate of accumulation. Hence it follows that either an increase in $s_{p}$ or $\pi$ decreases $\lambda^{*}$ in the short run. The contractionary effect on aggregate demand caused by either an increase in the saving propensity or a redistribution of income away from wages lowers the rate of technical progress through the Kaldor-Verdoorn effect. We may thus conclude that the productivity regime also turns out to be wage-led.

\section{MEDIUM-RUN EQUILIBRIUM}

For analytical tractability, in this paper we define a short, medium and long run, according to which variables are adjusting and which are not. We assume that the short-run position of the model economy is achieved by the adjustments of the rate of utilization of capacity $u$, while holding both the autonomous consumption to capital stock ratio $z$ and the animal spirits $\gamma$ as constant. Now, in the case of the medium run, we allow for changes in $z$ while still taking $\gamma$ as given.

We assume that $Z$ increases at the exogenously given rate of $\bar{g}_{z}$ over time. This way the Sraffian supermultiplier mechanism can be embedded into the model. Then $z$ evolves according to the following law of motion:

$$
\frac{\dot{z}}{z}=\bar{g}_{z}-g^{*}
$$

Using (7) and rearranging, we have:

$$
\frac{\dot{z}}{z}=\bar{g}_{z}-\frac{\gamma-\gamma_{u} u_{n}+\gamma_{\lambda} \lambda_{0}}{1-\gamma_{\lambda} \lambda_{g}}-\frac{\gamma_{u}}{1-\gamma_{\lambda} \lambda_{g}} u^{*}
$$

As shown with the help of (11), we can observe that the evolution of $z$ follows a stable path owing to the short-run stability condition (9). In other words, the same condition guarantees stability in both the short and medium run.

$$
\frac{\partial}{\partial z}\left(\frac{\dot{z}}{z}\right)=-\frac{\gamma_{u}}{1-\gamma_{\lambda} \lambda_{g}} \frac{\partial u^{*}}{\partial z}=-\frac{\gamma_{u}}{s_{p} \pi\left(1-\gamma_{\lambda} \lambda_{g}\right)-\gamma_{u}}<0
$$

It is natural to define the equilibrium condition in the medium run as:

$$
\dot{z}=0 \text {, }
$$


with $z>0$. Plugging (6) and (10) into (12), we can solve for the medium-run equilibrium value of $z$ :

$$
z^{* *}=\frac{\bar{g}_{z}\left[s_{p} \pi\left(1-\gamma_{\lambda} \lambda_{g}\right)-\gamma_{u}\right]-s_{p} \pi\left(\gamma-\gamma_{u} u_{n}+\gamma_{\lambda} \lambda_{0}\right)}{\gamma_{u}},
$$

where $* *$ denotes the value of a variable in the medium-run equilibrium. It can be noted that for $z^{* *}$ to be positive $\bar{g}_{z}$ must be large enough, as is argued in Lavoie (2017, p. 196):

$$
\bar{g}_{z}>\frac{s_{p} \pi\left(\gamma-\gamma_{u} u_{n}+\gamma_{\lambda} \lambda_{0}\right)}{s_{p} \pi\left(1-\gamma_{\lambda} \lambda_{g}\right)-\gamma_{u}}
$$

Also we have:

$$
\begin{gathered}
g^{* *}=\bar{g}_{z} \\
\lambda^{* *}=\lambda_{0}+\lambda_{g} \bar{g}_{z} \equiv \bar{\lambda} \\
u^{* *}=u_{n}+\frac{\bar{g}_{z}\left(1-\gamma_{\lambda} \lambda_{g}\right)-\gamma-\gamma_{\lambda} \lambda_{0}}{\gamma_{u}} .
\end{gathered}
$$

We can evaluate the medium-run effects of an increase in either $s_{p}$ or $\pi$. From (13) and (16), it follows that

$$
\begin{gathered}
\frac{\partial z^{* *}}{\partial s_{p}}=\pi u^{* *}>0 \\
\frac{\partial z^{* *}}{\partial \pi}=s_{p} u^{* *}>0 .
\end{gathered}
$$

This medium-run adjustment to an increase in $s_{p}$ or $\pi$ can be graphically illustrated as shown in Figure $1 .^{1}$ Assume our economy is initially at a medium-run equilibrium. Once the share of profits increases, the rate of accumulation goes down in the short run. However, when we move beyond the short run, the curve representing equation (10) gets flattened and shifts up. At first $z$ increases because the rate of accumulation, that is, the growth rate of the capital stock, falls short of that of autonomous consumption. This is reflected in the small arrow in the upper panel of Figure 1. Now since $\dot{z}>0$ at the current value of $z$, which is $z_{0}^{* *}, z$ starts to increase along the new line, say to $z_{1}^{* *}$. Then this in turn shifts down the saving curve from $\sigma_{1}\left(z_{0}^{* *}\right)$ to $\sigma_{1}\left(z_{1}^{* *}\right)$. At the new medium-run equilibrium, the rate of capacity utilization, the rate of accumulation, and the rate of technical progress simply come back to their initial positions.

The changes in the functional distribution of income do not affect the medium-run position of the economy if we compare only the initial and final positions. However, when we consider the whole traverse of the economy between the initial and the final position responding to a relevant change, it is apparent that non-neutral effects emerge

1. From now on we will omit the impact of changes in $s_{p}$ since they are symmetric to those in $\pi$. 


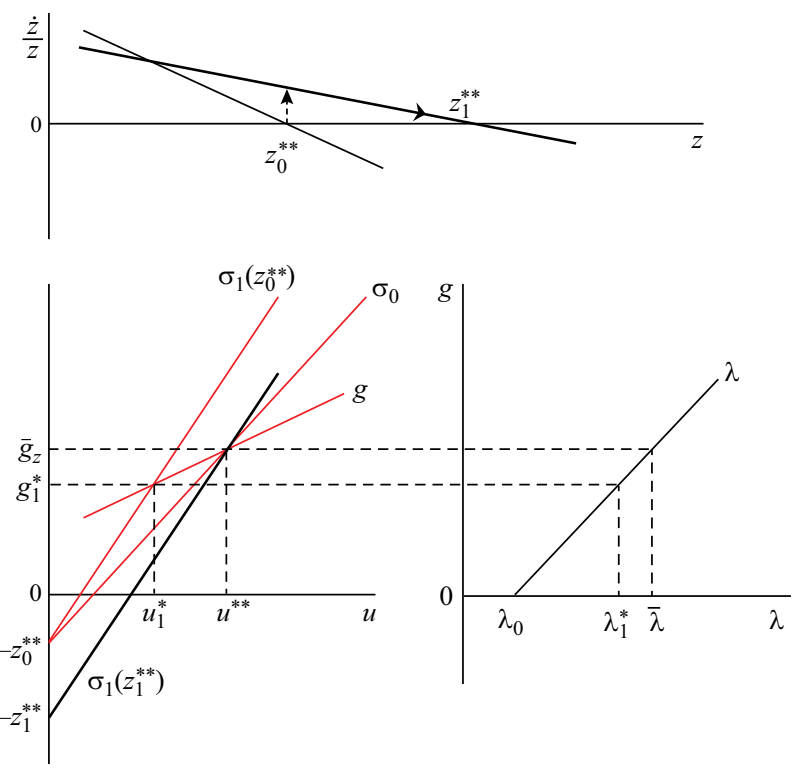

Figure 1 Medium-run adjustments to an increase in $s_{p}$ or $\pi$

on the average rate of accumulation, the average rate of capacity utilization, the average growth rate of labor productivity, and hence on both the level of capital stock and the level of labor productivity. This is surely consistent with some of the main tenets of the Keynesian perspective. In the case considered above, the redistribution of income favoring capital has a negative impact on average values without having an impact on the steady-state values achieved in the medium run.

Then what about the effects of an increase in $\bar{g}_{z}$ ? In the medium run, the line representing $\dot{z} / z=0$ shifts up in a parallel fashion. The result is an increase in $z^{* *}$. Note that $z$ increases whenever the average growth rate of the capital stock is lower than $\bar{g}_{z}$. However, when $\bar{g}_{z}$ itself increases, an increase in $z$ does not imply a lower growth rate of the capital stock. Indeed, from (13), we have:

$$
\frac{\partial z^{* *}}{\partial \bar{g}_{z}}=\frac{s_{p} \pi\left(1-\gamma_{\lambda} \lambda_{g}\right)-\gamma_{u}}{\gamma_{u}}>0 .
$$

Comparing the initial and the new medium-run equilibrium, that is, after $z$ reaches $z_{1}^{* *}$, and checking equations (14), (15), and (16), we can verify that the rate of utilization of capacity, the rate of accumulation and the rate of technical progress are all positively affected by an exogenous increase in $\bar{g}_{z}$. With $z$ adjusting from $z_{0}^{* *}$ to $z_{1}^{* *}$, all the average rates of utilization, accumulation, and technical progress are also positively affected.

This can be illustrated with the help of Figure 2 . An increase in $\bar{g}_{z}$ from $\bar{g}_{z}^{0}$ to $\bar{g}_{z}^{1}$ brings about a bigger $z^{* *}$, which in turn shifts down the saving curve. The new medium-run equilibrium has higher rates of accumulation, capacity utilization, and technical progress, which is $\left(\bar{g}_{z}^{1}, u_{1}^{* *}, \bar{\lambda}_{1}\right)$ in the figure. This is true provided there is no adjustment to the $\gamma$ parameter, as we shall see next. 


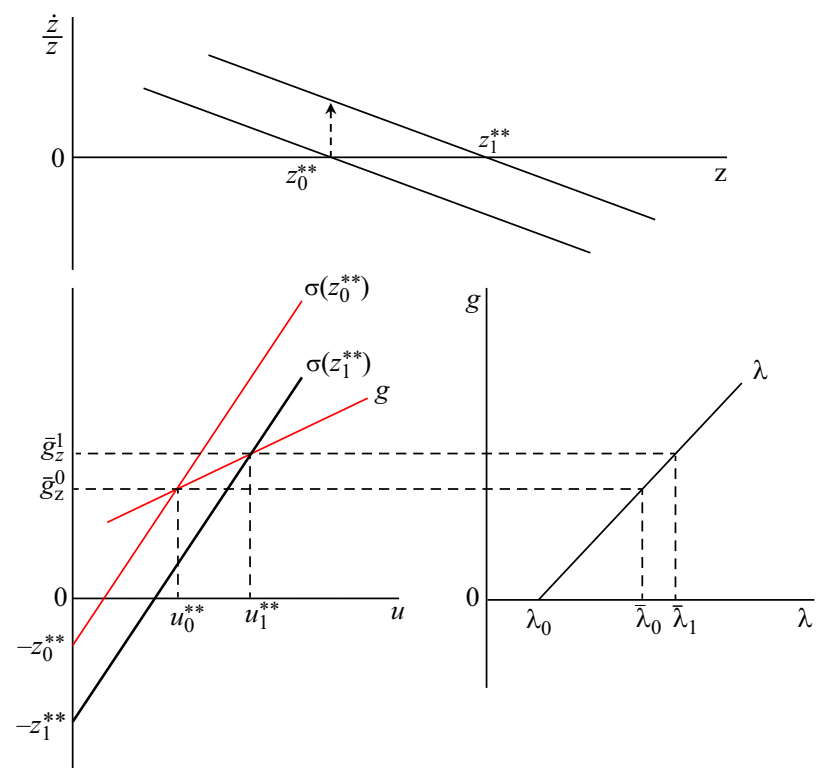

Figure 2 Medium-run effects of an increase in $\bar{g}_{z}$

\section{LONG-RUN EQUILIBRIUM}

It should be noted that in general the medium-run equilibrium rate of capacity utilization is not equal to its normal or target rate, $u^{* *} \neq u_{n}$. This means that the introduction of the Sraffian supermultiplier on its own does not bring our model to a fully adjusted position.

Now let us consider the longer run. Although Kalecki (1971, p. 183) objected to theories based 'on such fallacious a priori assumptions as a constant degree of long-run utilisation of equipment,' it is often claimed that in the long run, all the endogenous variables - including the rate of capacity utilization - should converge to their fully adjusted values. As was first shown by Allain (2015), somewhat paradoxically, the combination of an exogenous growth demand component with a Harrodian instability mechanism can bring about a convergence towards a fully adjusted position within a neo-Kaleckian model. To be specific, the long run is defined as the time period during which a Harrodian investment variable $\gamma$ in the accumulation function (3) changes together with $z$ and other endogenous variables in the present model. Notably, Harrodian instability occurs when the parameter $\gamma$ becomes a variable which goes up whenever firms are confronted with a demand which is unexpectedly high, and hence if the rate of accumulation accelerates to any level that exceeds the sum of both the expected rate of sales growth in the absence of technical progress, which is $\gamma$, and the expected rate of growth of capital purely induced by technical progress, which is $\gamma_{\lambda} \lambda$. Conversely, if the actual rate of accumulation is less than the expected growth rate of capital when technical change is taken into account, $\gamma$ goes down.

$$
\frac{\dot{\gamma}}{\gamma}=\psi\left(g^{*}-\gamma-\gamma_{\lambda} \lambda\right)
$$

where $\psi>0$ and $\gamma>0$. 
We interpret $\gamma$, which is a proxy for animal spirits - the sentiments and willingness among capitalists to invest - to be the expected rate of growth of capital when the effects of technical progress are ruled out. Here, because of the specification of our investment function as given by (3), it should be pointed out that $\gamma$ cannot be understood as the expected or trend growth rate of sales in the presence of technical change, as is the case in a number of neo-Kaleckian models which do not have explicitly considered technical change. In this regard, $\gamma$ must instead be only indirectly related to the growth rate of sales.

Straightforwardly from (3) we must have $\psi\left(g^{*}-\gamma-\gamma_{\lambda} \lambda\right)=\phi_{1}\left(u^{*}-u_{n}\right)$ with $\phi_{1}=\psi \gamma_{u}>0$. Putting this together with equations (17) and (6), we have:

$$
\frac{\dot{\gamma}}{\gamma}=\phi_{1}\left[\frac{\gamma+\gamma_{\lambda} \lambda_{0}+\left(1-\gamma_{\lambda} \lambda_{g}\right) z-s_{p} \pi u_{n}\left(1-\gamma_{\lambda} \lambda_{g}\right)}{s_{p} \pi\left(1-\gamma_{\lambda} \lambda_{g}\right)-\gamma_{u}}\right] .
$$

Using this Harrodian equation, we can form a Jacobian which governs the dynamics of the present system in a slightly modified manner, as found in Lavoie $(2014 ; 2016)$ :

$$
J=\left[\begin{array}{ll}
\frac{\partial(\dot{\gamma} / \gamma)}{\partial \gamma} & \frac{\partial(\dot{\gamma} / \gamma)}{\partial z} \\
\frac{\partial(\dot{z} / z)}{\partial \gamma} & \frac{\partial(\dot{z} / z)}{\partial z}
\end{array}\right]=\left[\begin{array}{cc}
\frac{\phi_{1}}{s_{p} \pi\left(1-\gamma_{\lambda} \lambda_{g}\right)-\gamma_{u}} & \frac{\phi_{1}\left(1-\gamma_{\lambda} \lambda_{g}\right)}{s_{p} \pi\left(1-\gamma_{\lambda} \lambda_{g}\right)-\gamma_{u}} \\
\frac{-s_{p} \pi}{s_{p} \pi\left(1-\gamma_{\lambda} \lambda_{g}\right)-\gamma_{u}} & \frac{-\gamma_{u}}{s_{p} \pi\left(1-\gamma_{\lambda} \lambda_{g}\right)-\gamma_{u}}
\end{array}\right]
$$

The determinant and the trace are reduced to:

$$
\begin{gathered}
\operatorname{Det} J=\frac{\phi_{1}}{s_{p} \pi\left(1-\gamma_{\lambda} \lambda_{g}\right)-\gamma_{u}}>0 \\
\operatorname{Tr} J=\frac{\phi_{1}-\gamma_{u}}{s_{p} \pi\left(1-\gamma_{\lambda} \lambda_{g}\right)-\gamma_{u}} .
\end{gathered}
$$

For this dynamic system to exhibit stability around its long-run equilibrium, DetJ should be positive and $T r J$ negative. We can see that the conditions for long-run stability in the model are fairly weak. The conditions are that: (i) the short-run Keynesian stability condition given in (9) should be met; and (ii) $0<\phi_{1}<\gamma_{u}{ }^{2}$ Here, (ii) implies that Harrodian instability should be existent but not too severe, so that it can be tamed by the stabilizing forces of the co-evolution of $z$ in the model, that is, $\partial(\dot{\gamma} / \gamma) / \partial \gamma>0$, and $\partial(\dot{\gamma} / \gamma) / \partial \gamma+\partial(\dot{z} / z) / \partial z<0$.

Finally, our long-run equilibrium can be easily derived as follows:

$$
\begin{gathered}
g^{* * *}=\bar{g}_{z} \\
\lambda^{* * *}=\lambda_{0}+\lambda_{g} \bar{g}_{z}=\bar{\lambda} \\
u^{* * *}=u_{n} \\
\gamma^{* * *}=\bar{g}_{z}\left(1-\gamma_{\lambda} \lambda_{g}\right)-\gamma_{\lambda} \lambda_{0}
\end{gathered}
$$

2. Because $\phi_{1}=\psi \gamma_{u}$, the second condition means that $0<\psi<1$. 
284 Review of Keynesian Economics, Vol. 7 No. 3

$$
z^{* * *}=s_{p} \pi u_{n}-\bar{g}_{z}
$$

where $* * *$ denotes the value of a variable in the long-run equilibrium. It should be noted that we must assume the following inequality to have a meaningful long-run equilibrium. Otherwise, $z^{* * *}$ may take on non-positive values.

$$
\bar{g}_{z}<s_{p} \pi u_{n}
$$

We can examine the dynamics of the system using a phase diagram defined on the $(z, \gamma)$ space. From (18) and (10), we can derive two demarcation lines where $\dot{\gamma} / \gamma=0$ and $\dot{z} / z=0$, respectively, as follows:

$$
\begin{gathered}
\frac{\dot{\gamma}}{\gamma}=0: \gamma=-\left(1-\gamma_{\lambda} \lambda_{g}\right) z+s_{p} \pi u_{n}\left(1-\gamma_{\lambda} \lambda_{g}\right)-\gamma_{\lambda} \lambda_{0} \\
\frac{\dot{z}}{z}=0: \gamma=-\left(\frac{\gamma_{u}}{s_{p} \pi}\right) z+\bar{g}_{z}\left(1-\gamma_{\lambda} \lambda_{g}-\frac{\gamma_{u}}{s_{p} \pi}\right)+\gamma_{u} u_{n}-\gamma_{\lambda} \lambda_{0} .
\end{gathered}
$$

It can be shown that the absolute value of the slope of the demarcation line for a constant $\gamma$, which is $\left(1-\gamma_{\lambda} \lambda_{g}\right)$, exceeds the one for a constant $z$, which is given by $\gamma_{u} / s_{p} \pi$, as long as the short-run stability condition (9) is fulfilled. Thus, the demarcation line for a constant $\gamma$ is steeper than the one for a constant $z$. Also the vertical intercept of the demarcation line for a constant $\gamma$ must exceed the one for a constant $z$, since

$$
\begin{gathered}
s_{p} \pi u_{n}\left(1-\gamma_{\lambda} \lambda_{g}\right)-\bar{g}_{z}\left(1-\gamma_{\lambda} \lambda_{g}-\frac{\gamma_{u}}{s_{p} \pi}\right)-\gamma_{u} u_{n} \\
=\frac{z^{* * *}}{s_{p} \pi}\left[s_{p} \pi\left(1-\gamma_{\lambda} \lambda_{g}\right)-\gamma_{u}\right]>0,
\end{gathered}
$$

again due to (9). Now we can illustrate the long-run co-evolution of $z$ and $\gamma$ using Figure 3 , where the long-run equilibrium of our model is given by point $E$, which is unique and stable.

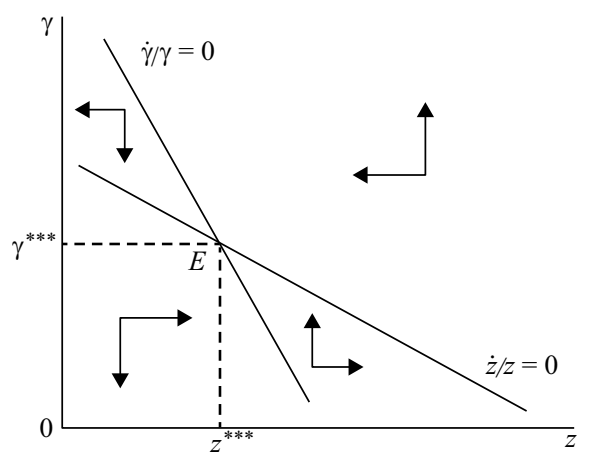

Figure 3 Phase diagram: co-evolution of $z$ and $\gamma$ 
Most of the discussions about comparative analyses for the medium run carry over to the long run, as can be verified by checking equations (19) to (23). Clearly an increase in the share of profits, $\pi$, leads to an increase in the long-run value of the autonomous consumption-to-capital-stock ratio, $z^{* * *}$. However none of the long-run rates of accumulation, capacity utilization, and technical progress are affected by the increase in the share of profits, while all of the average rates of accumulation, capacity utilization, and technical progress are lower during the traverse towards the fully adjusted position. In this sense, we can say that some of the main features of the Keynesian model are still valid, not only in the short run but also in the long run.

We can also analyse the long-run effects of a permanent increase in $\bar{g}_{z}$. Suppose the growth rate of autonomous consumption increases from $\bar{g}_{z}^{0}$ to $\bar{g}_{z}^{1}$. In Figure 3, this would shift up the demarcation line for a constant $z$ in parallel fashion, while there is no change in the other demarcation line. ${ }^{3}$ It is clear from (22) and (23) that in the new fully adjusted position $\gamma^{* * *}$ is larger while $z^{* * *}$ is smaller.

At first, an increase in the growth rate of autonomous consumption decreases saving by capitalists, which increases the rates of utilization, accumulation, and technical progress in the medium run. This adjustment process is represented by the gray downward arrow in Figure 4.

However, if we consider the longer time period, this should not be the whole story because now capacity is overutilized, at a rate which exceeds the target level. As a consequence, capitalists adjust their expectations, and this change in animal spirits in turn yields an upward shift of the accumulation curve. Moreover, this increase in the rate of accumulation pushes down the ratio of autonomous consumption to capital stock, again shifting the saving curve upward. The effects of this Harrodian adjustment are represented by the two black upward arrows in Figure 4.

Despite the fact that the rate of utilization is brought back to its normal value, the long-run rates of accumulation and of technical progress take on a permanent higher value. As to the rate of utilization, all that can be said is that its average value during the traverse is higher than the normal rate of utilization.

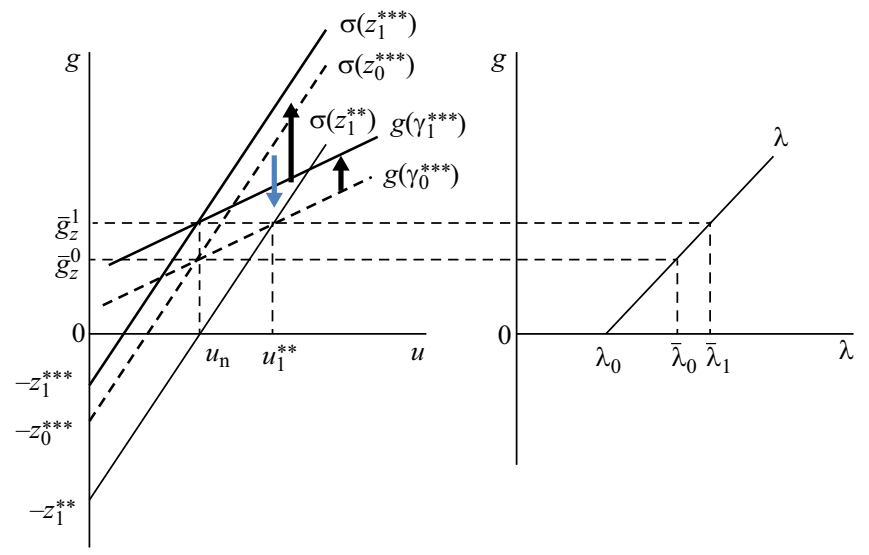

Figure 4 The long-run effects of an increase in $\bar{g}_{z}$

3. What then occurs in the phase diagram is identical to what is shown in fig. 5 of Lavoie (2016, p. 189). 


\section{THE EFFECTS OF PRODUCTIVITY SHIFTS}

In their assessment of growth models based on autonomous demand components, Trezzini and Palumbo (2016, pp. 515-516) insist that it is important to take shifts in technical progress into account. Let us then examine the effects of an exogenous technological shift, that is, an increase in the growth rate of technical progress which presumably does not arise from the introduction of new machines. This is represented by an increase in $\lambda_{0}$ in our model. As is evident from (6), (7), and (8), there are positive effects on the rates of utilization of capacity, capital accumulation, and technical progress in the short run. Graphically, this change shifts the investment curve up, from $g_{0}$ to $g_{1}$, and the productivity curve to the right both in a parallel fashion, as in Figure 5, so that in the short run there is an increase in the rate of utilization, the rate of accumulation, and the rate of technical progress.

$$
\begin{gathered}
\frac{\partial u^{*}}{\partial \lambda_{0}}=\frac{\gamma_{\lambda}}{s_{p} \pi\left(1-\gamma_{\lambda} \lambda_{g}\right)-\gamma_{u}}>0 \\
\frac{\partial g^{*}}{\partial \lambda_{0}}=\frac{\gamma_{u}}{1-\gamma_{\lambda} \lambda_{g}} \frac{\partial u^{*}}{\partial \lambda_{0}}+\frac{\gamma_{\lambda}}{1-\gamma_{\lambda} \lambda_{g}}>0 \\
\frac{\partial \lambda^{*}}{\partial \lambda_{0}}=1+\lambda_{g} \frac{\partial g^{*}}{\partial \lambda_{0}}>0
\end{gathered}
$$

Moving on to the medium run, we should consider the effects on $z$, the autonomous consumption-to-capital-stock ratio. From (13), we see that

$$
\frac{\partial z^{* *}}{\partial \lambda_{0}}=-\frac{s_{p} \pi \gamma_{\lambda}}{\gamma_{u}}<0
$$

Let us assume that the economy was initially at $\left(z_{0}^{* *}, u_{0}^{* *}, g_{0}^{* *}, \lambda_{0}^{* *}\right)$ in Figure 5. After an increase in $\lambda_{0}$, the equilibrium changes to $\left(z_{0}^{* *}, u_{1}^{*}, g_{1}^{*}, \lambda_{1}^{*}\right)$ at the intersection of $g_{1}$ and $\sigma_{0}$ in the short run. However, due to this increase in the rate of accumulation, $z$ decreases over time from $z_{0}^{* *}$ to $z_{1}^{* *}$. The adjustments in $z$ stop as soon as the rate

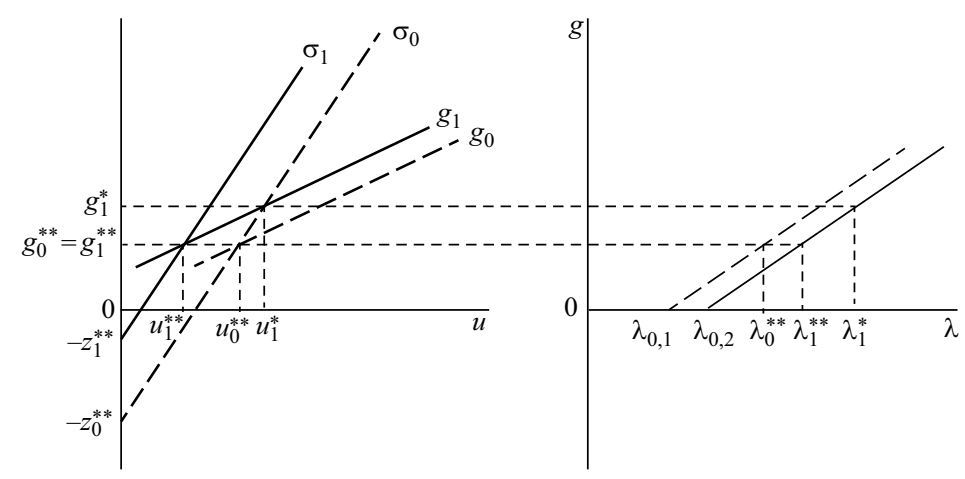

Figure 5 The medium-run effects of an exogenous improvement in productivity growth 
of accumulation becomes $g_{1}^{* *}=g_{0}^{* *}=\bar{g}_{z}$ unless $\bar{g}_{z}$, which is exogenously given, itself changes. Now the changes in $z$, in turn, shift up the saving curve from $\sigma_{0}$ to $\sigma_{1}$. A new medium-run equilibrium is reached at $\left(z_{1}^{* *}, u_{1}^{* *}, g_{1}^{* *}, \lambda_{1}^{* *}\right)$.

Apparently, there exist negative effects on the rate of capacity utilization, as can be confirmed algebraically from (16), and graphically from Figure 5.

$$
\frac{\partial u^{* *}}{\partial \lambda_{0}}=-\frac{\gamma_{\lambda}}{\gamma_{u}}<0
$$

The effects on the rates of both accumulation and technical progress need more consideration. Algebraically, from (14) and (15), we can see that an increase in $\lambda_{0}$ leads to no change in the medium-run rate of accumulation, $g^{* *}$, and a one-to-one increase in the rate of technical progress, $\lambda^{* *}$.

Thus, once more, comparing only the initial and terminal values of the rate of accumulation, we see that there is no change at all. However, when we consider the average values of the rate of accumulation during the whole duration after an exogenous increase in the growth rate of labor productivity in the medium run, we can conclude that there exist positive effects on the average rate of growth of the economy. Thus these results resemble those mentioned by Cesaratto et al. (2003, p. 49) in their discussion of the Sraffian supermultiplier mechanism, when they say that 'even when the effects of innovation on effective demand are positive, they are often level effects incapable of sustaining a higher growth rate of effective demand.'

The growth rate of labor productivity increases from $\lambda_{0}^{* *}$ to $\lambda_{1}^{*}$ in the short run. This is due to both the exogenous increase in $\lambda_{0}$ from $\lambda_{0,1}$ to $\lambda_{0,2}$ and the increase in the rate of accumulation from $g_{0}^{* *}$ to $g_{1}^{*}$. However, moving on to the medium run, while the actual rate of accumulation adjusts to the exogenously given growth rate of autonomous consumption, the short-run expansionary effects are contained and the rate of productivity growth decreases back to $\lambda_{1}^{* *}$, which is still at a higher value than before the change in $\lambda_{0}$. This is the reason why the medium-run rate of productivity growth falls short of the short-run one.

Finally, let us consider the long-run effects. In the long run, the negative mediumrun effect on the rate of capacity utilization is neutralized, and there is no effect left on the rates of either capacity utilization or accumulation, as is evident from (21) and (19). This is because the investment curve, which was shifted up in the medium run following the increase in productivity growth, comes back down to its original position while $\gamma$ adjusts to regain the normal rate of capacity utilization. All of this also occurs because the saving curve comes back down to its original position, reflecting the decrease in the rate of accumulation in the long run, as shown in Figure 6. This can also be seen by inspection of (23), which shows that $z^{* * *}$ is impervious to changes in the autonomous component $\lambda_{0}$ of technical progress, while $\lambda^{* * *}$ still has a one-toone relation to $\lambda_{0}$ (equation (20)).

In Figure 7, it is demonstrated that an increase in $\lambda_{0}$ shifts down both demarcation lines by the same amount, as is clearly seen from (25) and (26). Hence there is no change in the long-run position of $z$, while $\gamma$ decreases. The latter result can be derived by examining equation (22).

Readers may be puzzled by the belief held by Cesaratto et al. (2003), as well as the result obtained here, to the effect that an exogenous increase in the rate of technical progress will only have a transitory positive effect on the rate of accumulation. A contrario, to quote a recent paper, 'technical innovations may have a role in determining the pace of accumulation, something that has been stressed by many historians and 


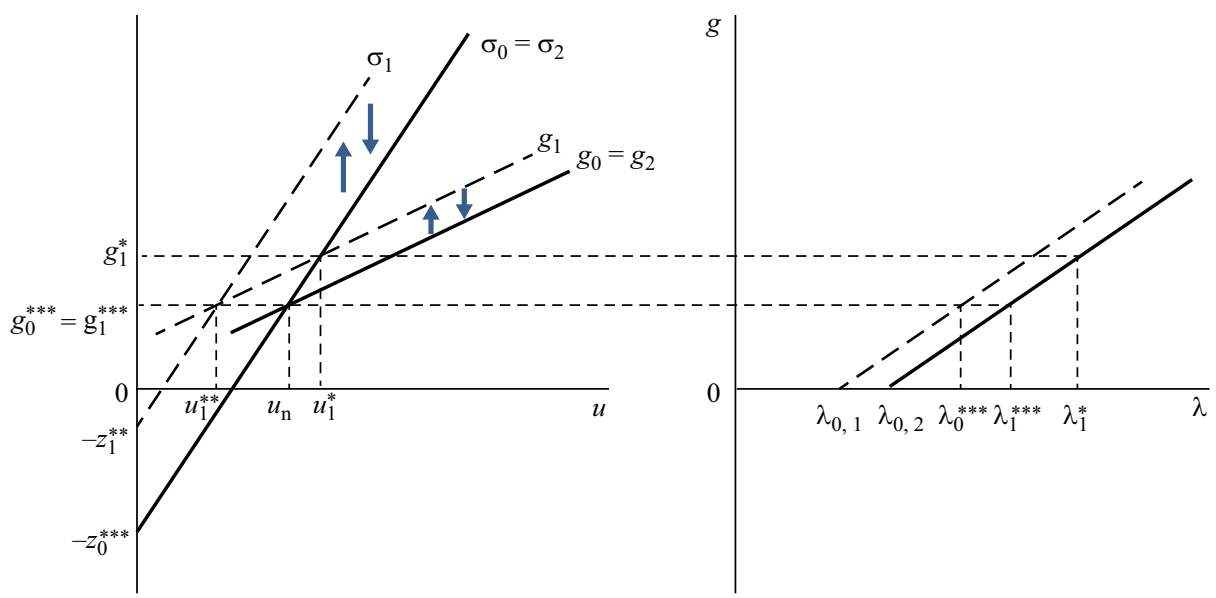

Figure 6 The long-run effects of an exogenous improvement in productivity growth

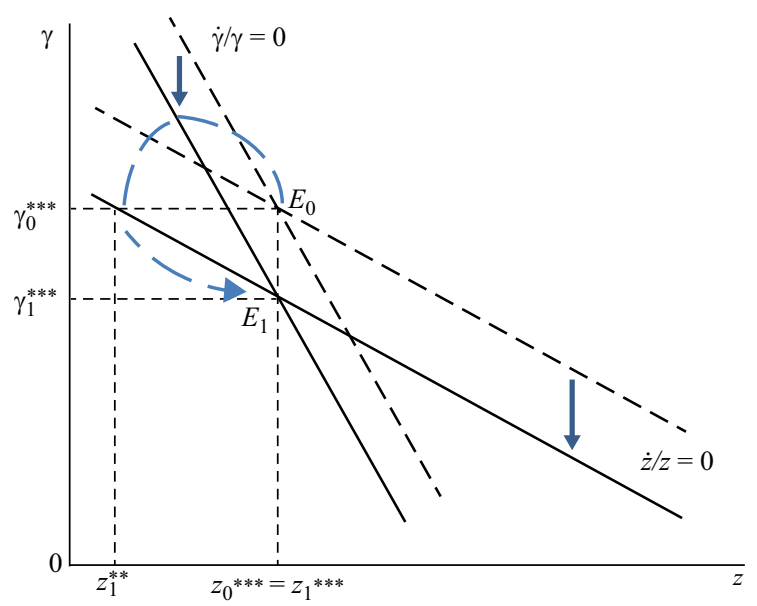

Figure 7 Co-evolution of $z$ and $\gamma$ after an exogenous improvement in productivity growth

economists in the analysis of historical processes of accumulation' (Trezzini and Palumbo 2016, p. 515). But as Dutt (2015, p. 35) points out, if we wish the rate of technological progress to help set the pace of long-run growth, 'technological change has to have some effect on aggregate demand in addition to its effect on investment.' This could occur, for instance, if faster technical change also generates an increase in the growth rate of the non-capacity-creating autonomous components of effective demand, for instance autonomous consumption, because of the accelerated pace in the introduction of novel and fashionable products. The very same possibility is raised by Dutt (ibid., p. 36) when he writes that one could 'interpret technological change as leading to product innovation and thereby determining the pace of autonomous 
capitalist consumption.' 4 This would imply making the 'autonomous' component of consumption a 'semi-autonomous component,' as Kalecki (1971, p. 174) would say, by writing it as a positive function of the rate of technical progress.

\section{IMPLICATIONS FOR EMPLOYMENT}

The present model provides additional implications for long-run employment, or rather the rate of growth of employment. Suppose our economy reaches its long-run equilibrium. Then the growth rate of output equals $\bar{g}_{z}$. Hence, in our model, the implied long-run growth rate of employment $g_{e}$ can be determined endogenously by subtracting the growth rate of labor productivity from the growth rate of output, as follows:

$$
g_{e}=\bar{g}_{z}-\left(\lambda_{0}+\lambda_{g} \bar{g}_{z}\right)=-\lambda_{0}+\left(1-\lambda_{g}\right) \bar{g}_{z}
$$

This relation implies that there exists a one-to-one relationship between the growth rate of employment and the growth rate of autonomous demand in the long run, with the latter determining the former, not the other way around. Moreover, we can say, provided the condition $\lambda_{g}<1$ is fulfilled, that there will be a positive relationship between the growth rate of employment and the growth rate of autonomous demand in the long run. As one would expect, this condition is likely to be satisfied, because, as reported by McCombie (2002, p. 106), estimates of the Kaldor-Verdoorn effect, that is, of $\lambda_{g}$, are in the 0.3 to 0.6 range, with more recent findings tending towards the former number. However, it can also be seen from the equation above that an increase in the autonomous component of technical progress (the part of technical progress which is not incorporated through investment) will lead to a one-to-one fall in the long-run growth rate of employment. This is the case despite the introduction in our investment function (3) of the assumption that technical progress induces faster accumulation. As noted above, this conclusion can only be evaded within this kind of model if we assume that faster technical change also generates an increase in the growth rate of the non-capacity-creating autonomous components of effective demand.

\section{CONCLUSION}

The Kaldor-Verdoorn law asserts that faster growth will induce faster technical progress. The idea that the recent productivity slowdown is an endogenous phenomenon reflecting the contraction in demand in the aftermath of the Great Recession seems to be getting wider acceptance. ${ }^{5}$ Along these lines, we have incorporated an endogenous technical progress function into a neo-Kaleckian model of growth with a non-capacity-creating autonomous growth component and the Harrodian instability mechanism.

It is demonstrated that the model converges towards the normal rate of capacity utilization in the long run under fairly weak conditions. However, even with this Classical feature, the main tenets of the Keynesian/Kaleckian model are shown to be valid, not

4. We do not believe, however, that it would be appropriate to write the investment equation as $g=\lambda+\gamma_{u}\left(u-u_{n}\right)$, for this would seem to reintroduce supply-side economics.

5. The idea that a slowdown in economic activity can cause a slowdown of the rate of technical progress, rather than the converse, can even be found in a recent article in The New York Times (see Irwin 2017). 
only in the short run but also in the long run. A decrease in the propensity to save or in the profit share will lead to faster growth during the transition towards the new long-run steady state, and hence to a higher average rate of growth during the traverse to the new fully adjusted position. In other words, one observes positive level effects when the propensity to save and the profit share are decreased. In addition, we have shown, under the same conditions, that the productivity regime is wage-led, meaning again that while the long-run value of the growth rate of productivity will not be modified by a decrease in the profit share, the average growth rate of productivity during the traverse to the new fully adjusted position will be higher when the wage share is permanently raised.

We have also examined what happens to the economy of our model when there is an increase in the growth rate of the autonomous component of consumption. Not surprisingly, such a change leads to an increase in the rate of accumulation and in the growth rate of labor productivity in both the medium and long run. Finally, we have examined the consequences of an increase in the exogenous component of technical progress. As pointed out informally by previous authors, such an increase only leads to a temporary increase in the rate of accumulation despite the higher long-run rate of technical progress. To avoid such a result, which at first hand would seem to be counter-intuitive, one would need to modify the core of the model and make the autonomous component of consumption receptive to the growth rate of technical progress and to innovations, thus rendering this component semi-autonomous.

\section{REFERENCES}

Allain, O. (2015), 'Tackling the instability of growth: a Kaleckian-Harrodian model with an autonomous expenditure component,' Cambridge Journal of Economics, 39(5), 1351-1371.

Allain, O. (2019), 'Demographic growth, Harrodian (in)stability and the supermultiplier,' Cambridge Journal of Economics, 43(1), 85-106.

Anzoategui, D., D. Comin, M. Gertler, and J. Martinez (2019), 'Endogenous technology adoption and R\&D as sources of business cycle persistence,' forthcoming in American Economic Journal: Macroeconomics.

Bortis, H. (1997), Institutions, Behaviour and Economic Theory: A Contribution to ClassicalKeynesian Political Economy, Cambridge, UK: Cambridge University Press.

Cesaratto, S. (2015), 'Neo-Kaleckian and Sraffian controversies on the theory of accumulation,' Review of Political Economy, 27(2), 154-182.

Cesaratto, S., F. Serrano, and A. Stirati (2003), 'Technical change, effective demand and employment,' Review of Political Economy, 15(1), 33-52.

Dejuán, O. (2017), 'Hidden links in the warranted rate of growth: the supermultiplier way out,' European Journal of the History of Economic Thought, 24(2), 369-394.

Dutt, A.K. (1990), Growth, Distribution and Uneven Development, Cambridge, UK: Cambridge University Press.

Dutt, A.K. (2015), 'Growth and distribution with exogenous autonomous demand growth and normal capacity utilization,' Mimeo, Notre Dame University.

Dutt, A.K. (2019), 'Some observations on models of growth and distribution with autonomous demand growth,' Metroeconomica, 70(2), 288-301.

Freitas, F. and F. Serrano (2015), 'Growth rate and level effects: the stability of the adjustment of capacity to demand and the Sraffian supermultiplier,' Review of Political Economy, 27(3), $258-281$.

Hein, E. (2018), 'Autonomous government expenditure growth, deficits, debt and distribution in a neo-Kaleckian growth model,' Journal of Post Keynesian Economics, 41(2), 316-338.

Irwin, N. (2017), 'Maybe we've been thinking about the productivity slowdown all wrong,' The New York Times, 25 July, available at: https://www.nytimes.com/2017/07/25/upshot/maybeweve-been-thinking-about-the-productivity-slump-all-wrong.html. 
Kaldor, N. (1957), 'A model of economic growth,' Economic Journal, 67(268), 591-624.

Kaldor, N. (1978), 'Causes of the slow rate of economic growth in the United Kingdom,' in N. Kaldor, Further Essays on Economic Theory, London: Duckworth, pp. 100-138.

Kalecki, M. (1971), Selected Essays in the Dynamics of the Capitalist Economy, Cambridge, UK: Cambridge University Press.

Lavoie, M. (1992), Foundations of Post-Keynesian Economic Analysis, Aldershot, UK and Brookfield, VT: Edward Elgar Publishing.

Lavoie, M. (2014), Post-Keynesian Economics: New Foundations, Cheltenham, UK and Northampton, MA: Edward Elgar Publishing.

Lavoie, M. (2016), 'Convergence towards the normal rate of capacity utilization in neo-Kaleckian models: the role of non-capacity creating autonomous expenditures,' Metroeconomica, 67(1), 172-201.

Lavoie, M. (2017), 'Prototypes, reality and the growth rate of autonomous consumption expenditures: a rejoinder,' Metroeconomica, 68(1), 194-199.

Mason, J.W. (2017), 'What recovery? The case for continued expansionary policy at the Fed,' The Roosevelt Institute, available at: http://rooseveltinstitute.org/wp-content/uploads/2017/ 07/Monetary-Policy-Report.pdf.

McCombie, J. (2002), 'Increasing returns and the Verdoorn law from a Kaldorian perspective,' in J. McCombie, M. Pugno, and B. Soro (eds), Productivity Growth and Economic Performance: Essays on Verdoorn's Law, Basingstoke, UK: Palgrave Macmillan, pp. 64-114.

Nah, W.J. and M. Lavoie (2017), 'Long-run convergence in a neo-Kaleckian open-economy model with autonomous export growth,' Journal of Post Keynesian Economics, 40(2), 223-238.

Nah, W.J. and M. Lavoie (2018), 'Overhead labour costs in a neo-Kaleckian growth model with autonomous expenditures,' IPE Working Papers 111/2018, Berlin School of Economics and Law, Institute for International Political Economy (IPE).

Nah, W.J. and M. Lavoie (2019), 'The role of autonomous demand growth in a neo-Kaleckian conflicting-claims framework,' Structural Change and Economic Dynamics, Advanced Access, available at: https://doi.org/10.1016/j.strueco.2019.02.001.

Pariboni, R. (2016a), 'Household consumer debt, endogenous money and growth: a supermultiplier-based analysis,' PSL Quarterly Review, 69(278), 211-233.

Pariboni, R. (2016b), 'Autonomous demand and the Marglin-Bhaduri model: a critical note,' Review of Keynesian Economics, 4(4), 409-428.

Reifschneider, D., W. Wascher, and D. Wilcox (2015), 'Aggregate supply in the United States,' IMF Economic Review, 63, 71-109.

Rowthorn, R.E. (1981), 'Demand, real wages and economic growth,' Thames Papers in Political Economy, Autumn, 1-39.

Serrano, F. (1995a), 'Long period effective demand and the Sraffian supermultiplier,' Contributions to Political Economy, 14, 67-90.

Serrano, F. (1995b), The Sraffian Multiplier, PhD dissertation, Faculty of Economics and Politics, University of Cambridge.

Serrano, F. and F. Freitas (2017), 'The Sraffian supermultiplier as an alternative closure to heterodox growth theory,' European Journal of Economics and Economic Policies: Intervention, 14(1), 70-91.

Steindl, J. (1979), 'Stagnation theory and stagnation policy,' Cambridge Journal of Economics, $3(1), 1-14$.

Trezzini, A. and A. Palumbo (2016), 'The theory of output in the modern classical approach: main principles and controversial issues,' Review of Keynesian Economics, 4(4), 503-522. 